\title{
Prevalence and interannual changes in multiple chemical sensitivity in Japanese workers
}

\author{
Xiaoyi Cui $\cdot$ Xi Lu $\cdot$ Mizue Hiura $\cdot$ \\ Masako Oda • Aya Hisada - Wataru Miyazaki • \\ Hisamitsu Omori $\cdot$ Takahiko Katoh
}

Received: 26 December 2013/Accepted: 14 January 2014/Published online: 30 January 2014

(C) The Japanese Society for Hygiene 2014

\begin{abstract}
Objective We aimed to evaluate the prevalence rates and interannual fluctuations in multiple chemical sensitivity (MCS) in Japanese workers.

Methods We assessed MCS using the Quick Environmental Exposure and Sensitivity Inventory, employing both Miller and Japanese criteria. Workers of two manufacturing companies located in Kyushu, Japan, were assessed, with company A surveyed in 2003, 2006 and 2011, and company B in 2003 and 2011.

Results In company A, the Miller criteria-based MCS prevalence rate was higher in 2011 than in 2003, and according to the Japanese criteria, it was higher in 2011 than 2006. In company B, the Miller criteria-based MCS prevalence rate was lower in 2011 than in 2003.

Conclusion The results indicated that MCS exists among industrial workers in Japan. We found no statistically significant interannual changes in MCS rates.
\end{abstract}

Keywords Multiple chemical sensitivity - Environmental exposure · QEESI · Sick house syndrome · Allergy · Japanese workers

X. Cui $(\bowtie) \cdot$ X. Lu · M. Hiura $\cdot$ M. Oda $\cdot$ A. Hisada .

W. Miyazaki $\cdot$ H. Omori - T. Katoh

Department of Public Health, Faculty of Life Sciences,

Kumamoto University, 1-1-1 Honjou, Chuo-ku,

Kumamoto 860-8556, Japan

e-mail: bjcxy860508@hotmail.com

H. Omori

Department of Biomedical Laboratory Sciences, Faculty of Life

Sciences, Kumamoto University, Kumamoto, Japan

\section{Introduction}

Multiple chemical sensitivity (MCS) is an acquired chronic disorder in which exposure to low levels of chemicals causes mild to wholly disabling symptoms [1]. Symptoms are usually vague and nonspecific, involving more than one organ system. In general, the reported symptoms are attributed to previous chemical exposure, and recur on subsequent exposure to chemicals at doses below those known to cause harmful effects in the general population [2]. The etiology of MCS, however, remains unclear. It is difficult to estimate its prevalence because it is derived from self-reports, which differ from case rates diagnosed by medical staff-occupational physicians in particular [1]. MCS patients' clinical characteristics are usually evaluated using questionnaires such as the Environmental Exposure and Sensitivity Inventory (EESI), or clinical interviews that rely on the individual's retrospective self-reports [3]. Miller and Prihoda [4] developed a globally standardized self-administered questionnaire, the Quick Environmental Exposure Sensitivity Inventory (QEESI), designed to assist researchers and clinicians in screening, studying, and evaluating patients with MCS.

People with environmental sensitivities may be susceptible to diverse environmental factors. Some of the more common agents containing chemical compounds that trigger reactions in such people include pesticides and volatile organic compounds (VOCs), such as solvents, perfumes, formaldehyde, and other petrochemicals. These agents may be contained in workplace building structures, furnishings, and cleaning products, among other sources [5]. A definition of work-related MCS was introduced by Cullen [6]. Adverse MCS health effects were observed in workers in subsequent research [7]; the negative effects of chemical hazards are a longstanding part of occupational 
health in the industrialized world [8]. While such issues initially tended to arise in industrial workers, similar problems have emerged in residents and workers in tight buildings in air- and water-polluted communities, and in persons exposed to various chemicals [9]. We selected workers employed in two large companies as participants, because this group allowed for easier follow-ups than community residents. The response rate may be higher from the workers who answered the questionnaires as instructed by company leaders. In addition to our aim of evaluating MCS prevalence rates and their interannual change, we assessed the rates of allergies and Sick house syndrome (SHS) in Japanese workers.

\section{Materials and methods}

\section{Study characteristics}

The present study was carried out at a paper pulp producing company (company A) in 2003, 2006, and 2011, and at an automotive company (company B) in 2003 and 2011, both in Kyushu, Japan. Subjects were asked to complete QEESI questionnaires, which also cover the respondents' diagnostic history of allergies and SHS. After excluding invalid questionnaires, we obtained 832 valid responses in 2003 , 729 in 2006, and 144 in 2011 at company A, and 333 responses in 2003 and 426 in 2011 at company B.

\section{Survey instruments}

We used the Japanese version of the QEESI questionnaire prepared by Ishikawa and Miyata to assess MCS [10]. Each criterion subscale of the QEESI contains 10 questions rated on a scale from 0 to 10 ; the total possible score for each subscale, therefore, ranges from 0 to 100 .

We used the Miller criteria to define MCS in workers according to the scores yielded by three cut-off subscales: $\geq 40$ for chemical sensitivity, $\geq 25$ for other chemicals, and $\geq 40$ for symptom severity classified as MCS [4]. Hojo et al. [11] designed a study to establish the cut-off value for Japanese criteria using the QEESI as an MCS screening method. We also employed their Japanese criteria of $\geq 40$ for chemical sensitivity, $\geq 20$ for symptom severity, and $\geq 10$ for life impact classified as MCS.

Data analysis

We collected and used anonymous information for data analysis. The distribution differences were examined using Chi square test. The average differences were examined using $t$ tests. Statistical analyses were carried out using SPSS version 18 for Windows (SPSS, Japan).
Ethical statement

The ethics review boards of Miyazaki University (no. 82; April 9, 2003) and Kumamoto University (no. 168; May 11, 2011) approved this study, following their ethical guidelines for human research. All participants provided written informed consent to participate, and the complete protection of their personal data was agreed upon in writing.

\section{Results}

At the 2003 baseline, SHS diagnostic history rates for companies $\mathrm{A}$ and $\mathrm{B}$ were 0.1 and $0.3 \%$, respectively, and the allergy diagnostic history rates for companies $\mathrm{A}$ and $\mathrm{B}$ were 23.1 and $24.0 \%$, respectively (Tables 1,2 ). The Miller-criteria-based MCS prevalence rate was $1.1 \%$ in company A and $2.4 \%$ in company B in 2003 (Figs. 1, 2).

In company A, SHS diagnostic history rate rose in 2011 (2.1\%) compared to 2003 and $2006(0.1 \%)$. The allergy diagnostic history rate decreased in 2006 (20.7\%) but rose in 2011 (39.6\%) (Table 1). In company B, the SHS and allergy diagnostic history rates $(0.5$ and $29.3 \%$, respectively) increased in 2011 (Table 2). In company A, the Miller-criteria-based MCS prevalence rate rose in 2011 (1.4\%) in comparison to 2003 and 2006 (1.1\%) (Fig. 1). However, in company B, Miller-criteria-based MCS prevalence rates dropped in 2011 (1.6\%) from $2003(2.4 \%)$ (Fig. 2).

As the life impact subscale was not employed in 2003, company A's QEESI-derived Japanese criteria-based MCS prevalence rate could only be determined between 2006 and 2011 (Fig. 3). The Japanese criteria-based MCS

Table 1 Diagnostic history and characteristics of respondents from company A

\begin{tabular}{llll}
\hline & 2003 & 2006 & 2011 \\
\hline $\begin{array}{l}\text { Diagnostic history, \% }(n) \\
\quad \text { Sick house } \\
\text { syndrome (SHS) }\end{array}$ & $0.1(1)$ & $0.1(1)$ & $2.1(3)$ \\
Allergy & $23.1(192)$ & $20.7(151)$ & $39.6(57)$ \\
None & $68.5(570)$ & $79.2(577)$ & $58.3(84)$ \\
No answer & $8.3(69)$ & $0(0)$ & $0(0)$ \\
$\quad$ Total & $100(832)$ & $100(729)$ & $100(144)$ \\
Sex, \% $(n)$ & & & \\
$\quad$ Female & $11.7(97)$ & $10.0(73)$ & $16.7(24)$ \\
$\quad$ Male & $88.3(735)$ & $90.0(656)$ & $83.3(120)$ \\
Total & $100(832)$ & $100(729)$ & $100(144)$ \\
Average age & $42.8 \pm 10.34$ & $44.9 \pm 10.61$ & $41.5 \pm 11.04$ \\
$\quad\left(\right.$ years \pm SD $\left.^{\mathrm{a}}\right)$ & & & \\
\hline
\end{tabular}

${ }^{a}$ Standard deviation 
prevalence rate increased in 2011 (4.2\%) from 2006 ( $3.3 \%)$; these prevalence rates were higher than those derived from the Miller criteria in 2011 (1.4\%) and 2006 $(1.1 \%)$ (Fig. 1). Nevertheless, these differences were not statistically significant.

In addition, the mean age of company $\mathrm{A}$ employees rose in 2006 (44.9 \pm 10.61 years old) and decreased in 2011 $(41.5 \pm 11.04$ years old) compared to baseline $(42.8 \pm 10.34$ years old $)$. The proportion of women in company A in $2011(16.7 \%)$ grew from $2003(11.7 \%)$ to $2006(10.0 \%)$ (Table 1). The mean age of employees in company B increased in 2011 (44.8 \pm 9.78 years old $)$

Table 2 Diagnostic history and characteristics of respondents from company B

\begin{tabular}{lll}
\hline & 2003 & 2011 \\
\hline $\begin{array}{l}\text { Diagnostic history, \% }(n) \\
\text { Sick house syndrome (SHS) }\end{array}$ & $0.3(1)$ & $0.5(2)$ \\
Allergy & $24.0(80)$ & $29.3(125)$ \\
None & $67.9(226)$ & $66.9(285)$ \\
No answer & $7.8(26)$ & $3.3(14)$ \\
$\quad$ Total & $100(333)$ & $100(426)$ \\
Sex, \% $(n)$ & & \\
Female & $0(0)$ & $3.1(13)$ \\
Male & $100(333)$ & $96.9(413)$ \\
Total & $100(333)$ & $100(426)$ \\
Average age (years $\left.\pm \mathrm{SD}^{\mathrm{a}}\right)$ & $40.5 \pm 8.65$ & $44.8 \pm 9.78$ \\
\hline
\end{tabular}

${ }^{a}$ Standard deviation when compared to 2003 s baseline ( $40.5 \pm 8.65$ years old); the proportion of females also grew from $2003(0 \%)$ to $2011(3.1 \%)$ (Table 2).

\section{Discussion}

This study investigated both Miller and Japanese criteriabased MCS prevalence rates across several years in Japanese general industrial workers.

Miller and Prihoda American study indicated that the MCS rate was $7.1 \%$ in 1999 [4]. A larger-scale investigation by Kreutzer et al. [12] employed a telephone survey, and found MCS rates of $6.3 \%$. In line with the present findings, studies by Uchiyama in 2000 and by Hojo in 2002 showed Japan's MCS rate to be lower than that of the USA $[13,14]$. In both companies, allergy diagnostic history rates rose in 2011 compared to 2003. Our findings were consistent with the increasing trend of allergy epidemic in Japan [15].

In the work environment, Watanabe et al. [16] identified several at-risk categories of chemicals, especially volatile compounds such as organic solvents. Many compounds used daily in manufacturing processes contained such chemicals, and exposed not only the workers who produced them, but also those in areas such as construction, automotive work, textiles, cleaning, and so on [1]. The impact of environmental sensitivities on workers may range from mild to severe, even making work impossible in some cases [5]. However, as this study's subjects were workers from

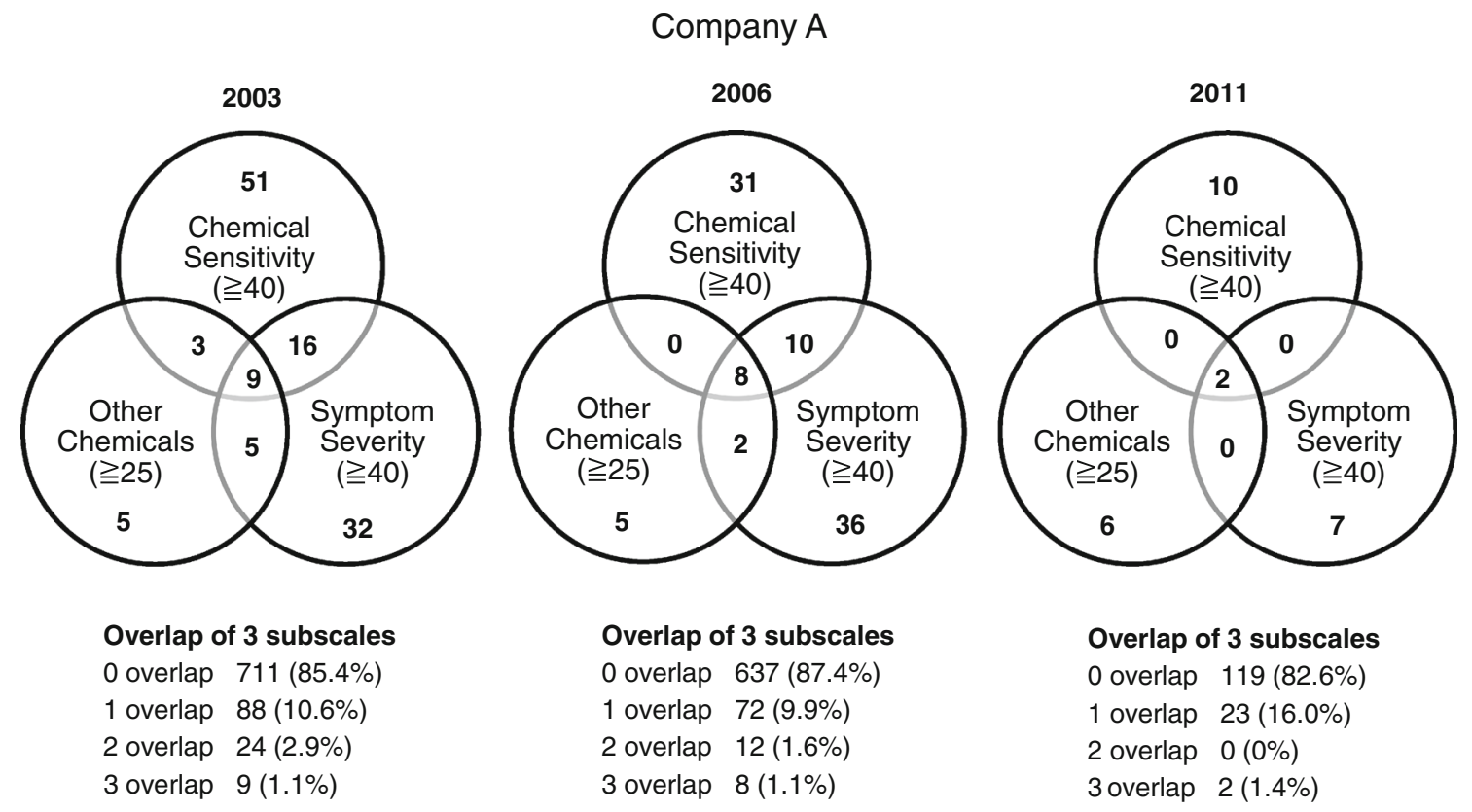

Fig. 1 Interannual changes in company A employee MCS rates, diagnosed through the QEESI using the Miller criteria (3 overlap was considered multiple chemical sensitivity) 
Fig. 2 Interannual changes in company B employee MCS rates, diagnosed through the QEESI using the Miller criteria (3 overlap was considered multiple chemical sensitivity)
Fig. 3 Interannual changes in company A employee MCS rates, diagnosed through the QEESI using the Japanese criteria (3 overlap was considered multiple chemical sensitivity)

\section{Company B}

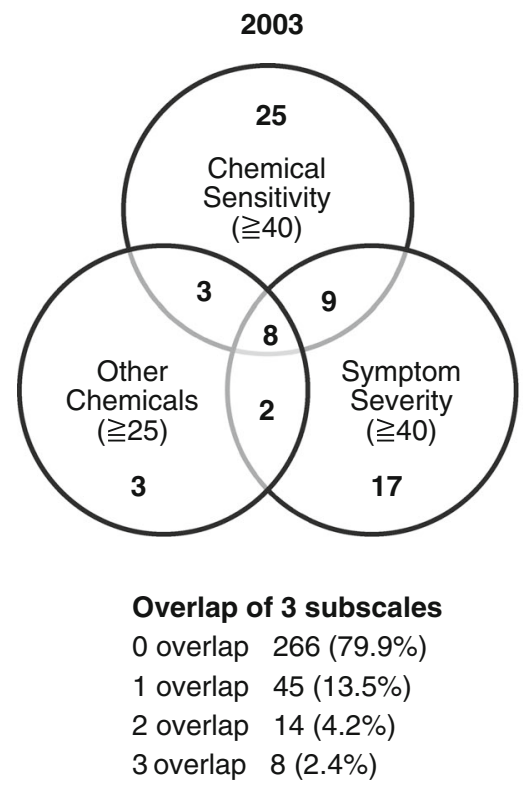

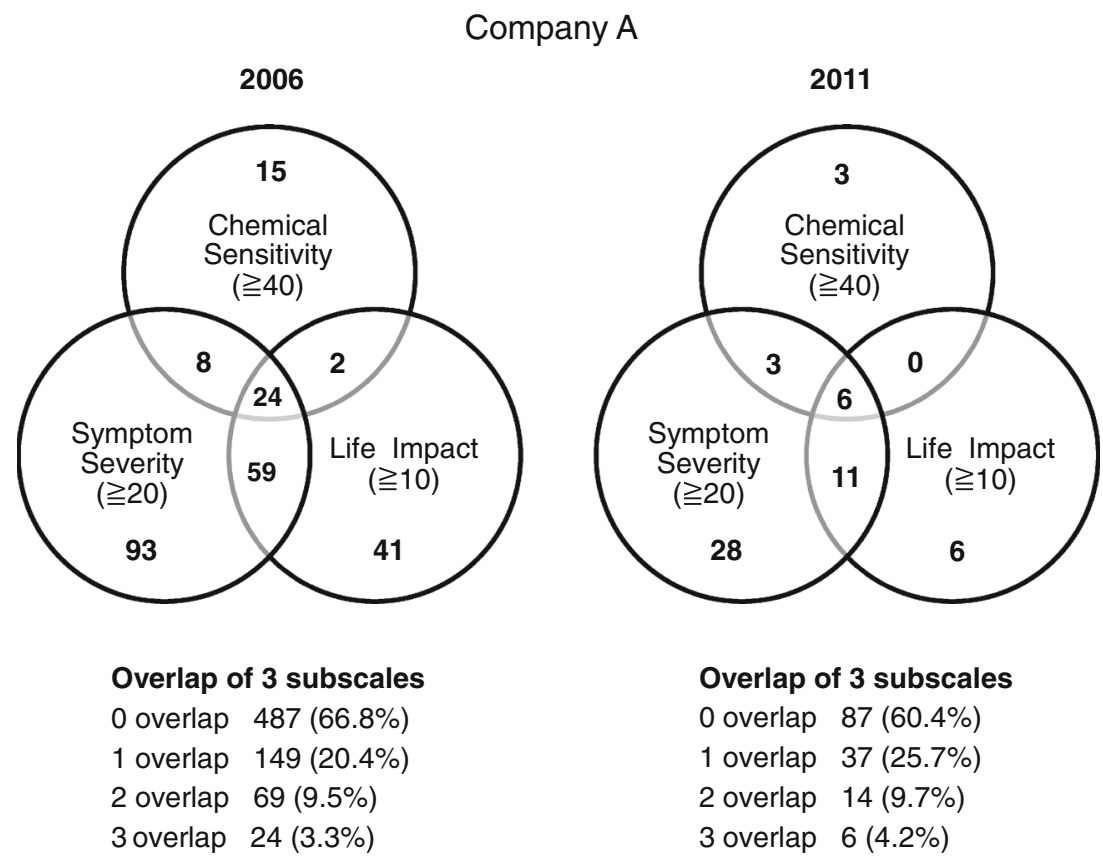

two large companies, it is important to note the selection bias known as the healthy worker effect (HWE), the process wherein unhealthy individuals are excluded from the workplace, as healthy workers are more likely to continue to work than those who are sick [17]. Some studies have suggested that incomplete follow-ups with workers who leave employment and migrate away from their workplace could be a source of the HWE $[18,19]$. Terr comments that some MCS patients change their jobs because of their symptoms [20], Lax and Henneberger later reported a similar conclusion [21]. A 2-year follow-up of 50 subjects with MCS showed that most were unchanged or worse by their final assessment [22]. Follow-up studies show that people with MCS frequently suffer symptoms for many years, but may show gradual improvement over time [16]. As the present study anonymized participants, it was impossible to confirm how many workers were continuously checked across several years. We also have found that it was impossible to follow-up with subjects who ceased working due to MCS. However, many patients were 
overlap in different years, which may indicate that they did not leave the workplace and were not recovered from MCS symptoms. The HWE may influence the MCS prevalence rate found in this study; however, this bias may not have had a significant impact on the results.

In occupational settings, exposures are often chronic. This suggests that controlling chemical exposure in the early phases of MCS may prevent more serious developments. Even for healthy people, numerous aggravating exposures may be below legal limits but not within adequate safety margins to prevent symptoms [23]. Improving environmental quality in the workplace can promote worker's health and productivity. Such workplace accommodation may include behavior changes, including the use of the least toxic cleaning products and pest control practices, and avoidance of scented products [5]. Furthermore, comparing estimated onset factors between male and female patients revealed that workplace chemical exposure was markedly higher in males [24].

Acknowledgments This work was supported in part by Grants-inAid for Research on Health Security Control (H24-Kenki-jpan) from the Ministry of Health, Labor and Welfare of Japan (http://www. mhlw.go.jp/). We express our sincere appreciation to the staff members of the Department of Public Health, Faculty of Life Sciences, Kumamoto University.

Conflict of interest No conflict of interest.

\section{References}

1. Martini A, Iavicoli S, Corso L. Multiple chemical sensitivity and the workplace: current position and need for an occupational health surveillance protocol. Oxid Med Cell Longev. 2013; 351457.

2. Graveling RA, Pilkington A, George JP, Butler MP, Tannahill SN. A review of multiple chemical sensitivity. Occup Environ Med. 1999;56:73-85.

3. Saito M, Kumano H, Yoshiuchi K, Kokubo N, Ohashi K, Yamamoto Y, et al. Symptom profile of multiple chemical sensitivity in actual life. Psychosom Med. 2005;67:318-25.

4. Miller CS, Prihoda TJ. The Environmental Exposure and Sensitivity Inventory (EESI): a standardized approach for measuring chemical intolerances for research and clinical applications. Toxicol Ind Health. 1999;15:370-85.

5. Sears ME. The medical perspective on environmental sensitivities. Can Hum Rights Commission. 2007;16-60.
6. Cullen MR. The worker with multiple chemical sensitivities: an overview. Occup Med. 1987;2:655-61.

7. Gibson PR. MCS: a survival guide. New Harbinger: Oakland; 2000.

8. Moen BE. Chemical sensitivity and the work place environment: research needs. Psychoneuroendocrinology. 2005;30:1039-42.

9. Ashford NA, Miller CS. Chemical exposures: low levels and high stakes. New York: Van Nostrand Reinhold; 1997.

10. Ishikawa S, Miyata M. Multiple chemical sensitivity-criteria and test methods for diagnosis. Allergol Immunol. 1999;6:990-8.

11. Hojo S, Sakabe K, Ishikawa S, Miyata M, Kumano H. Evaluation of subjective symptoms of Japanese patients with multiple chemical sensitivity using QEESI(c). Environ Health Prev Med. 2009; 14:267-75.

12. Kreutzer R, Neutra RR, Lashuay N. Prevalence of people reporting sensitivities to chemicals in a population-based survey. Am J Epidemiol. 1999;150:1-12.

13. Uchiyama I, Murayama R. Multiple chemical sensitivity as seen from the public health. In: Heisei 11 Welfare grant-in-aid for scientific research report. 2000. pp. 1-5 (in Japanese).

14. Hojyo S. Epidemiological study using QEESI in Japan. In: Heisei 13 Welfare grant-in-aid for scientific research report. 2002. pp. 134-152 (in Japanese).

15. Akasawa A. The development factors and medical system evaluation based on epidemiological study about national age prevalence of allergic diseases and treatment guidelines spread effect. In: Heisei 22 Labor and welfare grant-in-aid for scientific research report. 2011. pp. 245-250 (in Japanese).

16. Watanabe M, Tonori H, Aizawa Y. Multiple chemical sensitivity and idiopathic environmental intolerance (part two). Environ Health Prev Med. 2003;7:273-82.

17. Li CY, Sung FC. A review of the healthy worker effect in occupational epidemiology. Occup Med (Lond). 1999;49:225-9.

18. Monson RR. Observations on the healthy worker effect. J Occup Med. 1986;28:425-33.

19. Bell CM, Coleman DA. Models of the healthy worker effect in industrial cohorts. Stat Med. 1987;6:901-9.

20. Terr AI. Clinical ecology in the workplace. J Occup Med. 1989;31:257-61.

21. Lax MB, Henneberger PK. Patients with multiple chemical sensitivities in an occupational health clinic: presentation and follow-up. Arch Environ Health. 1995;50:425-31.

22. Terr AI. Environmental illness: a clinical review of 50 cases. Arch Intern Med. 1986;146:145-9.

23. Ziem G, McTamney J. Profile of patients with chemical injury and sensitivity. Environ Health Perspect. 1997;105:417-36.

24. Hojo S, Ishikawa S, Kumano H, Miyata M, Sakabe K. Clinical characteristics of physician-diagnosed patients with multiple chemical sensitivity in Japan. Int $\mathrm{J}$ Hyg Environ Health. 2008;211:682-9. 